\title{
Respect et violation des unités classiques dans la tragédie française du XIX ${ }^{\mathrm{e}}$ siècle
}

\section{Maurizio Melai}

\section{(2) OpenEdition}

\section{Journals}

\section{Édition électronique}

URL : http://journals.openedition.org/rief/937

DOI : 10.4000/rief.937

ISSN : 2240-7456

Éditeur

Seminario di filologia francese

\section{Référence électronique}

Maurizio Melai, « Respect et violation des unités classiques dans la tragédie française du XIX siècle », Revue italienne d'études françaises [En ligne], 1 | 2011, mis en ligne le 15 décembre 2011, consulté le 20 avril 2019. URL : http://journals.openedition.org/rief/937 ; DOI : 10.4000/rief.937

Ce document a été généré automatiquement le 20 avril 2019.

\section{(c) (i) (9)}

Les contenus de la RIEF sont mis à disposition selon les termes de la Licence Creative Commons Attribution - Pas d'Utilisation Commerciale - Pas de Modification 4.0 International. 


\title{
Respect et violation des unités classiques dans la tragédie française $\mathrm{du} \mathrm{XIX}^{\mathrm{e}}$ siècle
}

\author{
Maurizio Melai
}

1 Dès le début du XVIII ${ }^{e}$ siècle, les unités, piliers structurels de la tragédie classique, sont soumises, au moins autant que l'alexandrin, à de dures critiques. Ces contestations, toutefois, ne se concrétisent par des expériences théâtrales vraiment novatrices qu'à partir de 1759 , lorsque la scène est débarrassée de la présence encombrante du public ${ }^{1}$. C'est surtout la règle de l'unité de lieu qui est graduellement assouplie et contournée : le nouveau goût du spectacle visuel et des détails matériels de l'action, qui peut finalement s'exprimer sur une scène libre et spacieuse, se traduit par l'application de décors multiples dont l'usage est rendu systématique en particulier par De Belloy. Le principe de la concentration spatiale, assumant une souplesse aux traits vaguement préclassiques, en arrive à inclure dans ses marges de tolérance élargies les lieux adjacents d'un même palais ou d'une même ville².

2 Si l'Empire, avec ses velléités de purisme, favorise le retour à un respect plutôt strict des unités traditionnelles, la Restauration renoue avec l'expérimentation et avec la malléabilité normative de l'époque prérévolutionnaire. Partagée entre la fidélité aux conventions traditionnelles et l'expérimentation dramaturgique, la tragédie de l'époque post-napoléonienne entretient un rapport ambigu et controversé avec le bagage des contraintes formelles classiques, en particulier avec les règles des unités dramatiques. Les stratégies pour contourner les principes de l'unité de lieu et de temps, pour desserrer le nœud strict de l'unité d'action et pour fêler l'unité identitaire du héros tragique se font de plus en plus fines et raffinées, témoignant d'une intolérance croissante à l'égard du système normatif traditionnel. C'est sur ces stratégies que nous nous concentrerons dans cette brève étude.

3 Il faut d'abord souligner que les voix des défenseurs intransigeants des unités restent, tout au long des années 1820, les plus nombreuses et les plus influentes. À ce propos, nous pouvons citer l'affirmation emblématique du Journal du commerce, de politique et de 
littérature, qui fait du respect des unités une question de sauvegarde de l'honneur et du prestige culturel français : « Notre honneur national littéraire tient à la conservation des unités grecques " ${ }^{3}$, écrit cette revue le 10 août 1818. Le propos exprimé par le Journal des Débats le 13 mars 1820 est également significatif; en critiquant la conception spatiale de la Marie Stuart de Pierre Lebrun ${ }^{4}$, Étienne Becquet, feuilletoniste des Débats, soutient que "dès qu'on baisse la toile, ne fût-ce que pour passer de l'antichambre dans le salon, l'unité de lieu est totalement violée ». Plus encore que par les organes de presse, la cause de la résistance classique est soutenue par ces poètes qui, comme Népomucène Lemercier, tout en créant des situations tragiques d'une grande originalité, n'entendent pas dissocier ces contenus inédits du cadre traditionnel des unités. L'auteur de Frédégonde et Brunehaut, présentant sa pièce, déclare

avoir manifesté de nouveau, en observant le respect des trois unités grecques et latines, que les écarts et l'indépendance du genre nommé romantique ne produisent point d'émotions plus profondes et plus vives que n'en fournit la seule application exacte de nos classiques règles dans les mouvements passionnés artistement circonscrits en de sages limites. ${ }^{5}$

D'un autre côté, cependant, les critiques des réformateurs à l'égard des unités et des invraisemblances qu'elles produisent se font de plus en plus dures et pressantes. Le choix de Michel Pichat de situer le cinquième acte de son Léonidas ${ }^{6}$ dans les mêmes lieux que les quatre précédents et de ne pas déplacer la scène à Sparte, où le jeune Agis aurait dû informer les Grecs sur le sacrifice des trois cents, suscite une réaction véhémente de la part du globiste Duvergier de Hauranne, qui, le 14 janvier 1826, commente :

C'est à Sparte que se passait cet acte, à Sparte où nous attendait le tableau le plus intéressant. Au milieu de l'inquiétude générale, un guerrier arrivait couvert de sang et de poussière ; et ce laconique récit, «Ils sont tous morts, je meurs, » terminait dignement cette épopée dramatique. Croit-on que ce dénouement ne valût pas bien le songe de Xerxès et les horribles déclamations d'Archidamie? M. Pichat a craint sans doute de fatiguer l'imagination des classiques, et c'est pour aller au plus court qu'il nous a reconduits dans la tente de Xerxès.

De la même façon, dans un article non signé du 23 décembre 1824, le Globe s'en prend au Germanicus d'Arnault père ${ }^{7}$ pour «les langueurs des discours, qu'il eût été si facile de remplacer par des actions vives et naturelles, sans blesser en rien la règle si chère de l'unité de temps et de lieu »; « il n'eût fallu pour cela que consentir à sortir du palais ", ajoute le feuilletoniste. Il est intéressant d'observer que cette affirmation présuppose une conception des unités plutôt souple: tandis qu'Étienne Becquet considère le simple passage du héros tragique de l'antichambre au salon comme une infraction intolérable de l'unité spatiale, le journaliste du Globe ne voit aucune violation de la règle dans le déplacement des personnages en dehors du palais.

Cette divergence d'opinions entre les deux feuilletonistes est d'autant plus significative qu'elle dénonce le caractère aléatoire et discrétionnaire assumé par le concept d'unité pendant la Restauration : chacun en propose désormais sa définition, en en donnant une interprétation qui s'adapte à sa propre conception dramatique. De la querelle entre classiques et romantiques la plupart des dramaturges tirent une conception des unités, et en particulier de l'unité spatiale, parfaitement conforme à ce code intermédiaire qui caractérise la littérature tragique de l'époque. Dans un article du 14 janvier 1826, se plaignant de la médiocrité de la dramaturgie "semi-romantique», Duvergier de Hauranne commente ainsi l'évolution connue par les unités :

Voilà où en est aujourd'hui la lutte entre le catholicisme et le protestantisme littéraire; chacun propose sa petite transaction, et rien n'est plus curieux que 
d'entendre raisonner sur les unités ces semi-romantiques dont les salons de Paris commencent à se peupler. Ils avaient d'abord compris dans l'unité de lieu tous les appartements d'un même palais, puis tous les palais d'une même ville. Plus hardis aujourd'hui, une courte distance, comme, par exemple, celle de Paris à Saint-Cloud, ne les effraie pas trop.

7 En ce qui concerne l'unité de lieu, l'une des transactions les plus intéressantes est proposée par Lucien Arnault dans Pierre de Portugal. Critiqué pour avoir situé l'action en partie « à Lisbonne dans le palais des rois » et en partie «dans la campagne voisine », l'auteur justifie ses choix de la sorte :

Vingt-quatre heures sont fixées pour l'accomplissement d'une action dramatique. Ce laps de temps a paru la concession la plus forte que l'imagination du spectateur pût accorder à la durée d'événements qui, dans la réalité, s'achèvent en deux heures ; les entr'actes sont donc censés remplir l'espace qui n'est pas consacré à la portion visible du drame. Dans ces entr'actes, les personnages ne cessent pas d'agir et de se mouvoir ; pourquoi cette action et ce mouvement ne les conduiraient-ils pas d'un lieu à un autre? Pourquoi n'auraient-ils pas la faculté de parcourir une distance proportionnée au temps que notre imagination accorde à l'intervalle de deux actes ? Il me semble important qu'une action commence et se termine dans le même lieu, parce que cette condition est une garantie contre l'abus qu'on pourrait faire du déplacement permis aux personnages d'un drame. ${ }^{8}$

8 Certes, la possibilité d'un changement de lieu au cours des entractes et celle d'une unité garantie par la coïncidence entre le décor du premier acte et celui du dernier sont des idées plutôt communes à cette époque. Lucien Arnault, cependant, parvient à charger cette conception de l'espace dramatique d'implications stylistiques et idéologiques précises. Thématisant l'opposition spatiale entre «le palais des rois » de Lisbonne et «la maison très simple, entourée de bois " où vit Inès de Castro, l'auteur revendique la prérogative de varier son style pour mettre en scène des personnages appartenant à des classes sociales différentes. C'est ainsi qu'il se demande: "pourquoi, dans une composition destinée à reproduire un tableau des événements de la vie humaine, n'introduirait-on pas les contrastes de style nécessités par ceux des personnages, des sentiments et des situations? ${ }^{9}$ L'opposition spatiale se fait, en outre, dichotomie morale : la simplicité et l'honnêteté bourgeoise de l'habitation d'Inès contrastent avec le luxe d'un palais royal peuplé de courtisans dépravés et intrigants. Après un deuxième acte où la visite de Pierre chez Inès forme une parenthèse de bonheur amoureux à l'écart des fastes de la Cour, le déplacement d'Inès au palais royal correspond à un voyage vers l'échec et vers la mort: la jeune femme ne peut que se heurter aux préjugés d'une classe aristocratique qui refuse toute ingérence extérieure et qui, empêchant la montée sociale de l'héroïne bourgeoise, raffermit son autarcie inviolable. Dans ce sens, la circularité de la conception spatiale, garantie par le fait que l'action «commence et se termine dans le même lieu", semble surdéterminer au niveau structurel les idées d'immobilité et d'imperméabilité sociales contre lesquelles la pièce avance sa critique.

Si une telle mobilisation métaphorique de l'espace - déjà proche de celle qui sera effectuée par les romantiques ${ }^{10}$ - n'est certainement pas commune dans la dramaturgie tragique de la Restauration et de la Monarchie de Juillet, les nombreuses violations ou « réinterprétations extensives » de l'unité de lieu que l'on y relève répondent de manière générale à des exigences poétiques et à des critères expressifs bien définis. Le déplacement de la scène entre le premier et le deuxième acte, suivi d'un retour au décor initial dans l'acte final, est par exemple un procédé récurrent et riche en implications sémantiques. Parmi les auteurs qui en font un usage significatif, outre Lucien Arnault, 
nous pouvons mentionner Pierre Lebrun, qui s'en sert aussi bien dans sa Marie Stuart que dans son Cid d'Andalousie. Dans le premier cas, « la scène est en Angleterre (1587) au château de Fotheringay. Le premier et le cinquième acte se passent dans l'appartement de Marie; les autres, dans une salle ouverte de toutes parts sur les jardins de Fotheringay ». Si le passage à l'extérieur de l'appartement de Marie reflète le mouvement centrifuge vers la possibilité antitragique de la liberté et de la réconciliation, le retour à l'espace fermé initial représente la frustration de ce mouvement. Dans le cas du Cid d'Andalousie ${ }^{11}$, « la scène est à Séville, au XIII ${ }^{e}$ siècle (1284). Les I ${ }^{e r}$, III et $V^{e}$ actes se passent dans l'Alcazar, ancien palais des rois mores; le II et le IV dans les jardins et la maison de Doña Estrelle et de don Bustos ». Le déplacement vers l'habitation d'Estrelle incarne l'aspiration à un bonheur intime et privé, aspiration dont l'effondrement coïncide avec la reconduction des personnages vers les espaces du pouvoir.

Les passages de l'espace fermé à l'espace ouvert et vice versa sont souvent significatifs. Dans le sylla d'Étienne Jouy ${ }^{12}$, par exemple, «la scène se passe à Rome; dans les quatre premiers actes, dans le palais du dictateur; dans le cinquième, au Forum »: le déplacement de l'action vers la place publique, aussi bien dans ce cas que dans celui de la Virginie de Latour de Saint-Ybars ${ }^{13}$, symbolise la chute du despotisme et le retour du pouvoir dans les mains du peuple. Au contraire, dans un cas comme celui de l'Élisabeth de France d'Alexandre Soumet ${ }^{14}$, le passage des «rochers d'Aldovéra » au palais de Philippe d'Espagne traduit par des termes spatiaux le processus d'écrasement des opinions et des sentiments subi par les personnages.

La dialectique entre ouverture et fermeture de l'espace dramatique fait souvent en sorte, dans la tragédie des années 1820 , que l'action soit déplacée, d'un acte à l'autre, dans les différents palais et places d'une même ville. Plus que dans l'ensemble de la pièce, par conséquent, c'est à l'intérieur de chaque acte que l'intégrité du lieu de l'action doit être rigoureusement garantie: le changement de décor au cours des entractes devient une pratique extrêmement commune, qui a l'effet de transformer les actes en unités autonomes au niveau spatial. C'est ce qui arrive dans le Fiesque de Jacques Ancelot ${ }^{15}$, où l'on passe du palais de Fiesque au palais de Verrina et de celui-ci au palais ducal de Gênes, et encore dans le Marino Faliero de Casimir Delavigne ${ }^{16}$, où la scène se déplace respectivement du palais du doge à celui de Lioni et de ce dernier à la place Saint-Jean-etPaul. De même, dans la Jeanne d'Arc de Soumet ${ }^{17}$, les étapes du martyre de l'héroïne à Rouen coïncident avec autant de lieux emblématiques situés dans la ville : la prison, le palais de justice, la vieille place du palais de justice et enfin la grande place centrale où l'on dresse le bûcher.

12 À partir des dernières années de la Restauration, plusieurs auteurs dramatiques déplacent l'action tragique d'une région à l'autre et même d'un État à l'autre, franchissant la barrière spatiale constituée par les remparts de la ville : si Ancelot, par exemple, partage l'action d'olga entre la Petite-Tartarie et la Moscovie ${ }^{18}$, Pichat situe les différentes parties de l'intrigue de son Guillaume Tell dans les divers cantons suisses ${ }^{19}$. Dans les années 1840 , enfin, la volonté - plus prétendue que réelle - d'un retour au classicisme le plus pur n'empêche ni François Ponsard de partager la scène de sa Lucrèce entre Rome et Collatie ${ }^{20}$, ni Mme de Girardin de déplacer l'action de Cléopâtre d'Alexandrie à Tarente ${ }^{21}$. Toute sorte de déplacement du lieu de l'action est désormais virtuellement possible, pourvu que le changement de décor ne soit pas pratiqué au cours de l'acte, à l'intérieur duquel l'intégrité spatiale reste encore essentiellement inviolable. 
13 En ce qui concerne l'unité de temps, le code tragique de la Restauration et de la Monarchie de Juillet se maintient beaucoup plus près de la tradition. Certes, la mise en scène de plus en plus fréquente de faits d'histoire médiévale et moderne circonscrit l'intrigue dramatique dans une dimension temporelle bien déterminée; toutefois, à l'intérieur de cette dimension historique - définie souvent par l'indication explicite de l'année où les événements représentés se déroulent -, l'action tragique ne dépasse que rarement le cadre canonique des vingt-quatre heures.

Lorsque le déplacement spatial des protagonistes présuppose nécessairement l'infraction des vingt-quatre heures, comme dans les cas des voyages d'olga de la Petite-Tartarie à la Moscovie et de Cléopâtre de l'Égypte à l'Italie, le texte évite soigneusement de fournir au spectateur tout repère chronologique, ce qui confirme l'impression d'une extrême concentration temporelle des événements. Dans certains cas, comme dans le Cid d'Andalousie de Lebrun ou Les Enfants d'Édouard de Delavigne ${ }^{22}$, les indications de lumière révèlent que l'action se développe sur deux ou trois jours, ce qui semble constituer l'étendue temporelle maximale que l'action tragique puisse supporter : tout en mettant en scène l'Histoire, la tragédie se focalise sur un moment culminant et ponctuel du fait historique ; elle préfère se concentrer sur la crise et sur l'acmé dramatique précédant la catastrophe plutôt qu'illustrer l'événement dans sa globalité et dans toute son étendue spatio-temporelle. La seule exception à ce procédé, d'ailleurs très tardive, est probablement constituée par Charlotte Corday, où Ponsard se préoccupe d'insérer l'assassinat de Marat à l'intérieur d'un contexte historique et social qu'il traite amplement, montrant les causes et les conséquences du geste de l'héroïne ${ }^{23}$. Un dessin historiquement si vaste et si ambitieux ne peut se traduire que par la violation continuelle et systématique de l'unité de temps : prenant comme point de départ le 22 septembre 1792, à savoir le moment où «la République vient d'être proclamée par la Convention ", l'action se prolonge, passant par plusieurs étapes intermédiaires, jusqu'au 17 juillet 1793, date de l'exécution de Charlotte Corday.

Pour en revenir à la règle des vingt-quatre heures, généralement respectée par les auteurs tragiques de la Restauration et de la Monarchie de Juillet, il est nécessaire de remarquer que certains d'entre eux, tout en comprimant l'action à l'intérieur de cet espace temporel limité, parviennent à conférer aux notations d'heure et de temps une valeur sémantique pleine et inédite. Nous pouvons faire référence, à ce propos, à la scène du banc dans le Cid d'Andalousie, où la nuit participe activement des sentiments des amants, et encore par exemple au Vallia de Latour ${ }^{24}$, où le protagoniste décrit l'heure nocturne comme un allié précieux de ses desseins criminels. En attendant la tombée de la nuit, le duc des Goths se lance dans la tirade suivante (II, 6) :

La nuit !... Dans leurs forêts, sombre et vieille retraite,

Mes pères s'inspiraient de son horreur secrète ;

Les ténèbres jetaient dans ces cœurs indomptés

Des rêves de grandeur, de nobles voluptés,

Et ce fut d'une nuit orageuse et profonde

Que sortit leur projet de saccager le monde.

La nuit! De nos penchants elle irrite l'ardeur,

Elle nous affranchit d'une vaine pudeur,

Et de tous les objets les formes effacées

Ne laissent rayonner que nos seules pensées.

L'homme esclave au grand jour, dont l'éclat le poursuit,

Reprend la liberté dans l'ombre de la nuit.

La nuit ! De nos complots, fidèle confidente, 
Elle endort sous nos coups la victime imprudente.

Lorsqu'un homme est tombé le poignard dans le sein,

Elle emporte en fuyant le nom de l'assassin ;

Et si quelques regards interrogent son ombre,

Elle reste toujours silencieuse et sombre.

La nuit vient me servir, elle vient m'inspirer,

Et c'est avec la nuit que je vais conspirer.

Quant à l'unité d'action, quoique respectée en général, elle est ponctuellement minée par la création de scènes douées d'une certaine autonomie et non fonctionnelles à l'avancement de l'intrigue. Les principes structurels et génétiques de la tragédie du XIX siècle, moins conformes à un modèle fixe et plus complexes que ceux qui règlent la tragédie des siècles précédents, ne semblent pas toujours pouvoir être définis à partir du procédé traditionnel de la construction à rebours ${ }^{25}$. Des scènes telles que la célèbre "scène du banc" du Cid d'Andalousie ne sauraient s'accorder à la norme classique de l'organicité de chaque partie par rapport à l'ensemble de la composition dramatique. C'est pour cette raison, d'ailleurs, que le public du Théâtre-Français accueillit très sévèrement le tableau dramatique créé par Lebrun ; comme le rappelle Sainte-Beuve, «le parterre trouva qu'une telle scène était un hors-d'œuvre, qu'elle entravait la rapidité de l'action, en un mot, qu'elle violait ouvertement la règle, Semper ad eventum festina; il fut inexorable $»^{26}$. À cette même règle ne sauraient se conformer non plus les nombreuses scènes de « couronnement temporaire » que Lucien Arnault dissémine dans ses tragédies : au contraire, c'est justement grâce à leur caractère aléatoire et à leur excentricité par rapport au fil de l'action que ces scènes peuvent surdéterminer structurellement le message politique hautement contestataire de l'incongruité et de la précarité de la monarchie restaurée ${ }^{27}$.

De même, les différentes scènes « de boudoir » que l'on trouve dans l'Élisabeth d'Angleterre d'Ancelot ne paraissent pas participer directement au développement de la trame tragique ${ }^{28}$. Pierre Duviquet ne peut percevoir la scène où la comtesse de Suffolck exalte longuement les qualités des ouvrages shakespeariens que comme un excursus complètement superflu ; c'est ainsi qu'il écrit : «Quel rapport a-t-elle à la tragédie ? [...] M. Ancelot a cru utile de faire une profession de foi sur Shakespeare. C'est là tout le secret de la tirade ». Duviquet relève également la gratuité de la scène où les dames d'honneur examinent les étoffes précieuses qu'elles vont offrir à la reine; sur ce tableau de vie courtisane, il se demande: "Que fait cette petite galanterie domestique au terrible événement qui se prépare?» Ou encore, à propos de la scène où la reine essaie le bandeau qui lui est offert par la duchesse de Nottingham, le critique affirme : «On croit que cet incident aura des suites; il n'en est plus question. Était-ce la peine de distraire le spectateur par ce trait de coquetterie puérile ? [...] Tout cela [...] ralentit la marche de l'action $»^{29}$.

Les commentaires critiques de Duviquet sont l'expression emblématique de la perplexité des classiques intransigeants face à une tragédie moderne qui, aspirant à caractériser les personnages et le contexte où ils s'insèrent de manière plus nuancée et plus réaliste, s'attarde sur des détails de mœurs et de vie quotidienne au lieu de mener l'action droit au but. Cette nouvelle tendance contestée par les classiques se traduit significativement, à partir des années 1820 , par la tentative de création d'une "tragédie de caractère ", à savoir d'une tragédie qui tend plus à multiplier les situations faisant ressortir le caractère d'un personnage qu'à concentrer les effets d'une action unique et serrée. C'est Étienne Jouy qui, en 1821, avec son Sylla, revendique la paternité de ce nouveau genre de tragédie 
qui semblerait constitutivement incompatible avec les principes classiques de l'organicité et de la concaténation rigoureuse des scènes. Dans la préface qu'il met en tête de sa pièce, Jouy déclare avoir emprunté le premier cette voie de la tragédie de caractère que Corneille, Racine et Voltaire ont seulement entrevue et indiquée. "Séduit par l'idée que je tentais une route nouvelle - écrit-il - j'ai essayé de peindre, pour le théâtre, un des plus grands caractères qui aient étonné le monde $\aleph^{30}$. Insistant sur le caractère révolutionnaire de son ouvrage, il continue :

On avait jusqu'ici fait sortir du combat des passions, de la fatalité des événements, le pathétique et la terreur; j'ai essayé de les faire jaillir de la force d'un seul caractère, d'ouvrir au spectateur les abîmes du cœur chez un homme extraordinaire, et de tirer de là seulement tout l'intérêt de mon ouvrage. ${ }^{31}$ s'accompagne d'une construction du personnage extrêmement originale : rompant avec le principe classique de l'intégrité et de la cohérence du caractère tragique, l'auteur fait de Sylla un homo duplex, partagé entre les différentes instances qui composent sa personnalité ambiguë. Despote fatigué de l'être, tyran glorifiant la liberté, Sylla réunit les traits de plusieurs héros shakespeariens: s'il s'exclame «Mourir! Dormir enfin!» à l'instar d'Hamlet, il est persécuté dans le sommeil, à la manière de Macbeth, par les ombres de ses victimes criant vengeance (IV, 8). Présentant le dictateur comme un mélange de force et de faiblesse, de cruauté et de générosité, Jouy jette les bases d'une conception romantique du personnage, quitte à tenter de réduire la fracture du caractère tragique par une uniformité de style parfaitement classique.

21 Si Sylla ne descend jamais au comique, onze ans plus tard le Louis XI de Delavigne ${ }^{33}$, définissable aussi comme "tragédie de caractère ", franchit la barrière traditionnelle qui sépare les registres haut et bas pour construire un caractère plus ouvertement romantique, conçu comme mélange de tragique et de comique, de sublime et de grotesque. La nature ambiguë du roi Louis XI est décrite de la sorte par Commynes (I, 3) :

De vices, de vertus quel étrange assemblage !

Là, quel effroi honteux ! là, quel brillant courage !

Que de clémence alors, plus tard que de bourreaux !

Humble et fier, doux au peuple et dur aux grands vassaux,

Crédule et défiant, généreux et barbare,

Autant il fut prodigue, autant il fut avare.

Plus encore que l'unité et l'uniformité stylistiques, la pièce de Delavigne viole le principe de l'unité d'action, en se servant d'une série de situations dramatiques non étroitement fonctionnelles au développement de l'action. Louis XI est, comme l'écrit le Constitutionnel le 11 février 1832, « une tragédie de caractère [...], où l'action, secondaire, est appelée dans le seul but de faire ressortir les traits du principal personnage ». Le Journal des Débats en arrive jusqu'à affirmer, le même jour, que «le drame de M. Casimir Delavigne, n'est pas une tragédie. C'est, à proprement parler, une étude fort approfondie et curieusement, trop curieusement détaillée de Louis XI dans sa vieillesse ». En conduisant la tragédie sur la voie de l'étude de caractère, le dramaturge doit au moins partiellement desserrer le nœud strict de l'unité d'action, portant atteinte à la norme classique de la subordination des différentes situations dramatiques à l'égard du fil central de l'intrigue.

Revue italienne d'études françaises, 1 | 2011 
La violation de l'unité d'action est étroitement liée à la mise en discussion de l'unité et de la cohérence identitaire du caractère tragique ${ }^{34}$. Le personnage double typiquement shakespearien, qui montre seulement un côté de sa personnalité pour en cacher un autre et chez qui sont scindés pensée et actes, essence et apparence, s'insère progressivement dans la tragédie française à partir des années 1820. Outre Sylla et Louis XI, nous pouvons compter parmi les représentants de cette nouvelle typologie de personnage tragique prototype du héros du drame romantique - le Fiesque qu'Ancelot crée en 1824 en s'inspirant de Schiller, les personnages tragi-comiques de Marino Faliero et de Glocester que Delavigne emprunte respectivement, en 1829 et en 1833, à Byron et à Shakespeare, ou encore le Brutus mis en scène par Ponsard dans sa Lucrèce de 1843. Doués d'une nature double et présentant à la fois des traits héroïco-tragiques et des traits comiques et ridicules, ces personnages, tout en exprimant la crise du héros de la tradition classique, incarnent parfaitement les principes modernes de la perméabilité et de la coexistence des registres dramatiques. Il est intéressant de signaler, enfin, que dans certaines tragédies des années 1820 la thématique du dédoublement de l'identité se traduit par la création d'un personnage maudit et relégué aux marges de la société qui prélude au héros hugolien. C'est le cas de l'Idamore protagoniste du Paria de Delavigne ${ }^{35}$, qui se présente en affirmant : "Je foule un sol fatal à mes pieds interdit ; / Je suis un fugitif, un profane, un maudit... / Je suis un Paria... » (I, 1). Partagé entre son identité originelle de paria et sa nouvelle identité de chef des guerriers de Bénarès, Idamore incarne le parvenu contradictoire et refusé par une société hermétique, ou plutôt, métaphoriquement, la victime idéale de l'immobilisme social de la Restauration. En ce qui concerne la scission identitaire $\mathrm{du}$ personnage tragique, nous pouvons également mentionner le cas du caractère de Roger mis en scène par Charles Liadières dans son Conradin et Frédéric. Persécuté lui aussi par le retour d'un passé et d'une identité refoulés, il incarne, au même titre que l'Idamore de Delavigne, le héros fatal aux origines obscures que Victor Hugo développera quelques années plus tard. Le statut déjà « romantique » du personnage se fait évident dans ces vers qu'il prononce en s'adressant à Constance, la femme qu'il aime et qu'il devrait épouser (II, 6) :

[...] Sais-tu bien qui je suis?

Sais-tu que du destin victime infortunée,

Par lui, dès le berceau, ma vie est condamnée !

Que cet hymen funeste attirerait sur toi

Le fardeau des malheurs qui n'accablent que moi?

24 Il est nécessaire de préciser, toutefois, que Liadières met généralement en scène des personnages bien plus canoniques et traditionnels. Dans la préface de son Walstein publié en 1829, d'ailleurs, il répond aux reproches de cette partie de la critique qui a dénoncé « la physionomie un peu chevaleresque du principal personnage » en se demandant :

Est-ce dégrader un caractère que de le revêtir de couleurs dramatiques [...] ? [...] Walstein peint tout entier, ambitieux, jaloux, inquiet, superstitieux, décidé dans ses paroles, incertain dans ses actions, incapable de prendre un parti lorsqu'il a tout préparé pour le succès, serait-il un personnage dramatique ? ${ }^{36}$

À travers ces questions rhétoriques, Liadières défend une conception du "dramatique " extrêmement classique, condamnant de fait le Wallstein "tout entier" peint par Constant vingt ans plus tôt: malgré les nombreuses réformes du caractère tragique proposées par différents dramaturges au cours de la Restauration et de la Monarchie de Juillet, le principe de l'unité et de la cohérence du personnage continue de manière générale à prévaloir dans la tragédie de l'époque. Il est indéniable, cependant, d'après les 
réflexions et les exemples recueillis dans la présente étude, que ce principe, comme celui des trois unités pseudo-aristotéliciennes, s'assouplit progressivement pendant la période en question, se conformant aux exigences d'un code tragique où se mettent graduellement en place les ingrédients du drame romantique.

\section{NOTES}

1. Sur les tentatives d'expérimentation scénique qui précèdent 1759, voir R. Bret-Vitoz, L'Espace et la scène: dramaturgie de la tragédie française, 1691-1759, Oxford, Voltaire Foundation, 2008.

2. À ce propos, voir J.-P. Perchellet, L'Héritage classique. La tragédie entre 1680 et 1814, Paris, Champion, 2004, p. 145-148.

3. Précisons que le Journal du commerce, de politique et de littérature, qui paraît à partir de juillet 1817, devient le Constitutionnel : journal du commerce, politique et littéraire en mai 1819.

4. P. Lebrun, Marie Stuart, dans Euvres, Paris, Perrotin, 1844, t. I, p. 149-251 (première représentation de la pièce : Théâtre-Français, 6 mars 1820).

5. N. Lemercier, Préface de Frédégonde et Brunehaut, Paris, Barba, 1821, p.XII-XIII (première représentation de la pièce : Théâtre-Français, 27 mars 1821).

6. M. Pichat, Léonidas, Paris, Ponthieu, 1825 (première représentation: Théâtre-Français, 26 novembre 1825).

7. A.-V. Arnault, Germanicus, Paris, Chaumerot, 1817 (première représentation : Théâtre-Français, 22 mars 1817).

8. L. Arnault, Préface de Pierre de Portugal, dans Euvres dramatiques, Paris, Firmin Didot, 1865, vol. I, p. 178 (première représentation de la pièce : Théâtre-Français, 21 octobre 1823).

9. Ibid., p. 179-180.

10. Sur la valeur symbolique et métaphorique de l'espace dans la dramaturgie romantique, voir en particulier A. Ubersfeld, Le Roi et le Bouffon. Étude sur le théâtre de Hugo de 1830 à 1839, Paris, Librairie José Corti, 2001 (1 $1^{\text {re }}$ éd. 1973), p. 503-565.

11. P. Lebrun, Le Cid d'Andalousie, dans Euvres, cit., t. I, p. 255-418 (première représentation de la pièce : Théâtre-Français, $1^{\mathrm{er}}$ mars 1825).

12. É. Jouy, Sylla, Paris, Ponthieu, 1822 (première représentation : Théâtre-Français 27 décembre 1821).

13. I. de Latour, dit Latour de Saint-Ybars, Virginie, Paris, Tresse, 1845 (première représentation : Théâtre-Français 5 avril 1845).

14. A. Soumet, Élisabeth de France, Paris, Boucher, Delaforest, Barba, 1828 (première représentation: Théâtre-Français, 2 mai 1828).

15. J. Ancelot, Fiesque, Paris, Canel, 1824 (première représentation : Odéon, 5 novembre 1824).

16. C. Delavigne, Marino Faliero, dans Euvres complètes, Paris, Firmin Didot, 1877-1881 t. II, p. 1-109 (première représentation de la pièce : Porte Saint-Martin, 30 mai 1829).

17. A. Soumet, Jeanne d'Arc, Paris, Barba, 1825 (première représentation : Odéon, 14 mars 1825).

18. J. Ancelot, Olga, ou l'Orpheline moscovite, dans CEuvres complètes, Paris, Delloye et Lecou, 1838, p. 157-177.

19. Précisons que le Guillaume Tell de Michel Pichat, représenté pour la première fois à l'Odéon le 22 juillet 1830, n'est publié que quarante ans après sa création, dans un volume qui inclut les Euvres de Pichat (Paris, Savigné, 1870). 
20. F. Ponsard, Lucrèce, Paris, Furne, 1843 (première représentation : Odéon, 22 avril 1843).

21. D. de Girardin, Cléopâtre, Paris, Michel Lévy frères, 1847 (première représentation: ThéâtreFrançais, 13 novembre 1847).

22. C. Delavigne, Les Enfants d'Édouard, dans Euvres complètes, cit., t. II, p. 286-409.

23. F. Ponsard, Charlotte Corday, Paris, Blanchard, 1850 (première représentation: ThéâtreFrançais, 23 mars 1850).

24. I. de Latour, dit Latour de Saint-Ybars, Vallia, Paris, Tresse, 1841 (première représentation : Théâtre-Français, 27 septembre 1841).

25. Les enjeux de ce procédé ont été mis en évidence par G. Forestier dans Essai de génétique théâtrale : Corneille à l'œuvre, Paris, Klincksieck, « Collection d'esthétique », 1996.

26. Ch.-A. Sainte-Beuve, « Notice sur les ouvrages de M. Lebrun », dans P. Lebrun, Euvres, cit., t. I, p. XXX-XXXI.

27. Sur la situation dramatique du couronnement temporaire et sur l'usage significatif qu'en fait en particulier Lucien Arnault dans ses tragédies de la Restauration, voir M. Melai, «Couronnement factice et vérité morale : une scène emblématique de l'imaginaire tragique de la Restauration ", dans Orages. Littérature et culture 1760-1830, n 9, mars 2010, p. 324-338.

28. J. Ancelot, Élisabeth d'Angleterre, dans Euvres complètes, cit., p. 181-212.

29. Les trois citations sont tirées de P. Duviquet, Examen critique d'Élisabeth d'Angleterre, dans J. Ancelot, Euvres complètes, cit., p. 210.

30. É. Jouy, Préambule historique de Sylla, cit., p. XXV-XXVI.

31. Ibid., p. XXVI-XXVII.

32. Ibid., p. XXVI.

33. C. Delavigne, Louis XI, dans Euvres complètes, cit., t. II, p. 124-285 ( $1^{\text {re }}$ représentation : ThéâtreFrançais, 11 février 1832).

34. Dans la treizième leçon de son Cours de littérature dramatique, Schlegel voit la scission identitaire $d u$ personnage - scission introduite au théâtre par Shakespeare, «premier des peintres de caractère » - comme l'un des éléments qui distinguent les "théâtres romantiques » $\mathrm{du}$ «théâtre classique». Voir A. W. von Schlegel, Cours de littérature dramatique, traduit de l'allemand par Mme Necker de Saussure, Paris, A. Lacroix, Verboeckoven et Cie, 1865, vol. II, p. 158-177.

35. C. Delavigne, Le Paria, dans Euvres complètes, cit., t. I, p. 155-232 (première représentation de la pièce : Odéon, $1^{\mathrm{er}}$ décembre 1821).

36. Ch. Liadières, Préface de Walstein, Paris, Vente, 1829 (sans numéros de page). Première représentation de la pièce : Théâtre-Français, 22 octobre 1828.

\section{INDEX}

Mots-clés : tragédie néoclassique, règles classiques, unités dramatiques, bienséances, héros tragique 